\title{
Resistome in gram-negative bacteria from soft cheese in Brazil
}

\author{
Resistoma em bactérias gram-negativas de queijo minas frescal no Brazil
}

\author{
Cristiane Rodrigues Silva ${ }^{1}$, Newton Takeshi Okuno², Victor Hugo Lima de Medeiros Macedo ${ }^{3}$, Isabela da \\ Rocha Freire ${ }^{4}$, Rafael Monção Miller ${ }^{3}$, Victor Augustus Marin ${ }^{5 *}$
}

\begin{abstract}
${ }^{1}$ Mestre e Doutoranda em Alimentos e Nutrição do Programa de Pós-Graduação em Alimentos e Nutrição da Universidade Federal do Estado do Rio de Janeiro (UNIRIO); ${ }^{2}$ Mestre em Alimentos e Nutrição pelo Programa de Pós-Graduação em Alimentos e Nutrição da UNIRIO; ${ }^{3}$ Acadêmico do Curso de Graduação em Nutrição da UNIRIO; ${ }^{4}$ Nutricionista pela UNIRIO; ${ }^{5}$ Doutor em Biotecnologia Vegetal, Docente da Escola de Nutrição e dos Programas de Pós-Graduação em Alimentos e Nutrição e, em Segurança Alimentar e Nutricional da UNIRIO.
\end{abstract}

\begin{abstract}
Objective: evaluation of antibiotic resistance in Gram-negative microbiota from ready-to-eat cheese samples. Methodology: this research applied an adapted methodology to select from a food sample viable Gram-negative microbiota displaying antibiotic resistance. The selected food was a cheese that is commonly consumed without thermal processing, the Minas Frescal cheese. The evaluation was followed by a PCR screening in this resistant microbiota, for genes that provide resistance to antibiotics and also to the quaternary ammonium. Results: all cheese samples harbored a resistant microbiota. In 13.3\% of the cheese samples analyzed, the resistance reached all ten different antibiotics tested and, in $80 \%, 8$ to 10 different antibiotics. In antibiotics considered critics as the carbapenems: ertapenem presented resistant microbiota in $86.7 \%$ of the samples. In cephalosporins, the resistance reached $100 \%$ in the third generation (ceftazidime) and almost half of the samples (46.7\%) in the fourth generation (cefepime). In genotypic research, seven different resistance genes were found in $69.2 \%$ of the bacterial pools, including the beta-lactamase-producing genes ctx, tem, shv, tetracycline-resistant genes, and a high rate of integrons class 1 and 2. Conclusion: the results indicate phenotypically and genotypically that the Minas Frescal cheese can harbor potential resistant microbiota. Therefore, the methodology used is a viable possibility and with a broader answer about the food microbiota role in resistance. This research corroborates the food area as an important sector to be managed to reduce the process of antibiotic resistance.
\end{abstract}

Keywords: Antibiotic Resistance. Food. Resistome. Gram-negative bacteria. Cheese.

\begin{abstract}
Resumo
Objetivo: avaliação da resistência a antibióticos em microbiota Gram-negativa de amostras de queijo prontas para consumo. Metodologia: esta pesquisa aplicou uma metodologia adaptada para selecionar a microbiota Gram-negativa viável apresentando resistência a antibióticos em uma amostra de alimento. O alimento selecionado foi um queijo frequentemente consumido sem processamento térmico, o queijo Minas Frescal. A avaliação foi seguida de uma triagem por PCR, nesta microbiota resistente, para genes que fornecem resistência aos antibióticos e também ao quaternário de amônio. Resultados: todas as amostras de queijo apresentaram microbiota resistente. Em 13,3\% dos queijos analisados essa resistência alcançou todos os 10 diferentes antibióticos testados e em $80 \%$ entre 8 e 10 antibióticos diferentes. Em antibióticos considerados críticos como os carbapenêmicos: ertapenem apresentou microbiota resistente em $86,7 \%$ das amostras. Nas cefalosporinas, a resistência atingiu $100 \%$ na terceira geração (ceftazidima) e quase a metade das amostras $(46,7 \%)$ na quarta geração (cefepime). Na pesquisa genotípica, sete diferentes genes de resistência foram encontrados em $69,2 \%$ dos pools bacterianos, incluindo o genes produtores de beta-lactamase, genes de resistência à tetraciclina, ctx, tem, shv e uma alta taxa de integron classe 1 e 2. Conclusão: os resultados indicam fenotipicamente e genotipicamente que o queijo Minas Frescal pode apresentar uma potencial microbiota resistente. Portanto, a metodologia utilizada é uma possibilidade viável e com uma resposta mais ampla sobre o papel da microbiota na resistência. Esta pesquisa corrobora a área de alimentos como um setor importante a ser gerenciado para redução no processo de resistência a antibióticos.
\end{abstract}

Palavras-chave: Resistência a antibióticos. Alimento. Resistoma. Bactéria Gram-negativa. Queijo.

\section{INTRODUCTION}

Resistant bacteria can be found virtually everywhere: in the environment, humans, animals, water, and food. The

Correspondente/Corresponding: *Victor Augustus Marin - Laboratório de Controle Microbiológico de Alimentos da Escola de Nutrição (LACOMEN), Departamento de Ciência de Alimentos (DCA), Escola de Nutrição, Universidade Federal do Estado do Rio de Janeiro (UNIRIO). - End: Avenida Pasteur, 296, Urca - Rio de Janeiro/RJ - CEP.: 22290-240 - Tel: (21)2542-7276/7294 - E-mail: victor.marin@unirio.br latter, though, is often wrongly neglected (WHO, 2017). The One health approach highlights the need for coordination between human and animal surveillance systems aiming for an integrated strategy to limit the spread of resistance. The use of antibiotics in food-producing animals provides a potentially significant risk factor for the selection and dissemination of antimicrobial-resistant microorganisms to humans, which makes animal-derived food of particular concern (EFSA, 2017). 
In Brazil, cheese, a ready-to-eat dairy product, is widely consumed by the adult population and, above all, by the elderly population (IBGE, 2010). Minas Frescal cheese is a traditional Brazilian white and soft cheese with very high moisture content ( $\geq 55 \%$ ) made with pasteurized milk. It has its own rich and diverse microbiota that impacts its quality and safety (BRASIL, 1997). Moreover, they are usually consumed without any further thermal processing, including nosocomial patients (VICENSKI; ALBERTI; AMARAL, 2012).

The research of individual species underestimates the total microbial community and its potential antimicrobial resistance risks (ESCOBAR-ZEPEDA; SANCHEZ-FLORES; QUIRASCO BARUCH, 2016). Since a rapid phenotypic detection of resistance can prevent delays in selecting optimal therapy (AZIMI et al., 2013), in this study, an adapted methodology focused on evaluating the microbiota resistance profile in food rather than the isolation and identification of resistant species.

The World Health Organization recognized the multidrug and extensively drug-resistant Gram-negative bacteria as the most critical microorganisms that researches should focus on (WHO, 2017). In this work, we present an evaluation of the phenotypic resistance and the presence of antibiotic-resistant genes in Gram-negative bacteria population in Minas Frescal cheese available in retail stores in Rio de Janeiro city.

\section{METHODOLOGY}

\section{Sampling}

Fifteen Minas Frescal cheese samples from 13 different commercial brands were retrieved from fifteen supermarket chains in the city of Rio de Janeiro, Brazil. Only cheese samples in their original intact packaging with the surface temperature at low temperature (less than $8^{\circ} \mathrm{C}$ ) and containing the Federal or State Inspection Seal (sanitary certifications) were purchased. Samples were transported in a thermal container to the Laboratório de Controle Microbiológico de Alimentos da Escola de Nutrição (LACOMEN) of the Departamento de Ciência de Alimentos of the Universidade Federal do Estado do Rio de Janeiro (UNIRIO) no later than 24 hours after collection.

\section{Pre-selection method}

Each cheese sample $(25 \mathrm{~g})$ was incubated at $35^{\circ} \mathrm{C} \pm$ $0.2^{\circ} \mathrm{C}$ in $225 \mathrm{~mL}$ of Gram-negative (GN) broth (Oxoid, Basingstoke, UK) for 24 hours.

After this enrichment stage, aliquots of $50 \mu \mathrm{L}$ from each cheese (sample) were diluted in $5 \mathrm{~mL}$ of tryptone soy broth (TSB) (Oxoid, Basingstoke, UK) containing antibiotic discs (Oxoid, Basingstoke, UK) (CDC, 2008). This procedure was done in triplicate, generating three tubes for each antibiotic tested. The ten antibiotics used in testing were representative of Ambler Classification: Cefepime $(30 \mu \mathrm{g})$, Ertapenem $(10 \mu \mathrm{g})$, Gentamicin $(10 \mu \mathrm{g})$, Ampicillin $(10 \mu \mathrm{g})$,
Ampicillin-sulbactam (10/10 $\mu \mathrm{g})$, Chloramphenicol $(30 \mu \mathrm{g})$, Tetracycline (30 $\mu \mathrm{g})$, Ciprofloxacin $(5 \mu \mathrm{g})$, Ceftazidime (30 $\mu \mathrm{g})$, Trimethoprim-sulfamethoxazole (1.25/23.75 $\mu \mathrm{g})$.

After 18-24h of incubation, all TSB tubes with turbidity from microbial growth were considered positive and qualified for the next step.

\section{Antibiotic Resistance Selection}

An adapted disc diffusion method using Agar MacConkey based on the methodology of the guidelines from the Clinical and Laboratory Standards Institute (CLSI) for antimicrobial disk susceptibility test and the Laboratory Protocol for Detection of Carbapenem-Resistant or Carbapenemase-Producing Klebsiella spp. and Escherichia coli from Rectal Swabs was performed in order to select the Gram-negative resistant microbiota (CDC, 2008; CLSI, 2017). Each positive tube of the triplicate from the previous step was inoculated in an agar plate, conserving the triplicates.

According to breakpoints for Enterobacteriaceae, the bacterial pools that were classified as resistant or intermediate in agreement with the CLSI guidelines for the interpretation of antibiotic susceptibility were collected for DNA extraction (CLSI, 2017).

For the DNA extraction step and consequently for $P C R$, each positive agar plate from the same triplicate was blended to be considered a unique pool. This procedure does not interfere in the analysis as each pool represents the microbiota from tests with the same antibiotic and the same cheese. Therefore, this strategy means that 15 cheese samples tested with 10 antibiotics generate a maximum number of 150 pools, in case of $100 \%$ of resistance.

The reference strains used in this step were E. coli ATCC 25922.

\section{DNA extraction}

DNA was extracted from all bacterial pools considered resistant or intermediate by using a NucleoSpin Tissue kit (Macherey-Nagel, Germany, 2016) according to the manufacturer's protocol for DNA extraction from Gram-negative bacteria.

\section{PCR}

Molecular characterization of phenotypically screened bacterial pools was performed by PCR by using previously published primers and protocols (Table 1). Primers were synthesized by Eurofins Genomics (Ebersberg, Germany) and Invitrogen Thermo Fisher Scientific (California, USA).

$P C R$ screening for the primers described in table 1 was done in all resistant or intermediate pools except for the ctx-M1, ctx-M2, ctx-M8, ctx-M9, ctx-M15, and ctx-M25 that were done in bacterial pool harboring ctx-M. The oxa-48 gene (involved in resistance to carbapenems) was screened in pools resistant to carbapenems, and tet $a$ and tet $b$ genes screened in pools resistant to tetracycline antibiotics. 
Table 1 - Primers: sequence of nucleotides, amplicon size and references.

\begin{tabular}{|c|c|c|c|c|}
\hline Primer & Primer sequence $\left(5^{\prime}-3^{\prime}\right)$ & $\begin{array}{c}\text { Amplicon } \\
\left(b^{a}\right)\end{array}$ & PCR Conditions & Reference \\
\hline $\begin{array}{l}\operatorname{tem}(F)^{b} \\
\operatorname{tem}(R)^{b}\end{array}$ & $\begin{array}{l}\text { CATTTCCGTGTCGCCCTTATTC } \\
\text { CGTTCATCCATAGTTGCCTGAC }\end{array}$ & 800 & $\begin{array}{l}10 \mathrm{~min} \text { at } 94^{\circ} \mathrm{C} ; 30 \text { cycles of } 40 \mathrm{~s} \text { at } 94^{\circ} \mathrm{C} \text {, } \\
40 \mathrm{~s} \text { at } 60^{\circ} \mathrm{C}, 60 \mathrm{~s} \text { at } 72^{\circ} \mathrm{C} \text {; and } 7 \mathrm{~min} \text { final } \\
\text { extension at } 72^{\circ} \mathrm{C} \text {. }\end{array}$ & $\begin{array}{l}\text { (DALLENNE et al., } \\
\text { 2010) }\end{array}$ \\
\hline $\begin{array}{l}\operatorname{shv}(F)^{b} \\
\operatorname{shv}(R)^{b}\end{array}$ & $\begin{array}{l}\text { AGCCGCTTGAGCAAATTAAAC } \\
\text { ATCCCGCAGATAAATCACCA }\end{array}$ & 713 & $\begin{array}{l}10 \mathrm{~min} \text { at } 94^{\circ} \mathrm{C} ; 30 \text { cycles of } 40 \mathrm{~s} \text { at } 94^{\circ} \mathrm{C} \text {, } \\
40 \mathrm{~s} \text { at } 60^{\circ} \mathrm{C}, 60 \mathrm{~s} \text { at } 72^{\circ} \mathrm{C} \text {; and } 7 \mathrm{~min} \text { final } \\
\text { extension at } 72^{\circ} \mathrm{C} \text {. }\end{array}$ & $\begin{array}{l}\text { (DALLENNE et al., } \\
\text { 2010) }\end{array}$ \\
\hline $\begin{array}{l}\text { oxa }(F)^{b} \\
\text { oxa }(R)^{b}\end{array}$ & $\begin{array}{l}\text { GACCCCAAGTTTCCTGTAAGTG } \\
\text { GGCACCAGATTCAACTTTCAAG }\end{array}$ & 564 & $\begin{array}{l}10 \mathrm{~min} \text { at } 94^{\circ} \mathrm{C} ; 30 \text { cycles of } 40 \mathrm{~s} \text { at } 94^{\circ} \mathrm{C}, \\
40 \mathrm{~s} \text { at } 60^{\circ} \mathrm{C}, 60 \mathrm{~s} \text { at } 72^{\circ} \mathrm{C} \text {; and } 7 \mathrm{~min} \text { final } \\
\text { extension at } 72^{\circ} \mathrm{C} \text {. }\end{array}$ & $\begin{array}{l}\text { (DALLENNE et al., } \\
\text { 2010) }\end{array}$ \\
\hline $\begin{array}{l}\text { int }-1 \\
\text { (F) } \\
\text { int }-1 \\
\text { (R) }\end{array}$ & CTGCGTTCGGTCAAGGTTCT & 882 & $\begin{array}{l}3 \mathrm{~min} \text { at } 94^{\circ} \mathrm{C} ; 35 \mathrm{cycles} \text { of } 60 \mathrm{~s} \text { at } 94^{\circ} \mathrm{C} \text {, } \\
60 \mathrm{~s} \text { at } 68^{\circ} \mathrm{C}, 60 \mathrm{~s} \text { at } 72^{\circ} \mathrm{C} \text {; and } 7 \mathrm{~min} \text { final } \\
\text { extension at } 72^{\circ} \mathrm{C} \text {. }\end{array}$ & $\begin{array}{l}\text { (LANZ; KUHNERT; BO- } \\
\text { ERLIN, 2003) }\end{array}$ \\
\hline $\begin{array}{l}\text { int - } 2 \\
\text { (F) } \\
\text { int }-2(R)\end{array}$ & $\begin{array}{l}\text { CACGGATATGCGACAAAAAGG } \\
\text { GTAGCAAACGAGTGACGAAATG }\end{array}$ & 788 & $\begin{array}{l}3 \mathrm{~min} \text { at } 94^{\circ} \mathrm{C} ; 35 \mathrm{cycles} \text { of } 60 \mathrm{~s} \text { at } 94^{\circ} \mathrm{C}, \\
60 \mathrm{~s} \text { at } 68^{\circ} \mathrm{C}, 60 \mathrm{~s} \text { at } 72^{\circ} \mathrm{C} \text {; and } 7 \mathrm{~min} \text { final } \\
\text { extension at } 72^{\circ} \mathrm{C} \text {. }\end{array}$ & $\begin{array}{l}\text { (LANZ; KUHNERT; BO- } \\
\text { ERLIN, 2003) }\end{array}$ \\
\hline $\begin{array}{l}\text { int }-3 \\
\text { (F) } \\
\text { int - } 3(R)\end{array}$ & $\begin{array}{l}\text { GCCTCCGGCAGCGACTTTCAG } \\
\text { ACGGATCTGCCAAACCTGACT }\end{array}$ & 979 & $\begin{array}{l}3 \mathrm{~min} \text { at } 94^{\circ} \mathrm{C} ; 35 \mathrm{cycles} \text { of } 60 \mathrm{~s} \text { at } 94^{\circ} \mathrm{C} \text {, } \\
60 \mathrm{~s} \text { at } 68^{\circ} \mathrm{C}, 60 \mathrm{~s} \text { at } 72^{\circ} \mathrm{C} \text {; and } 7 \mathrm{~min} \text { final } \\
\text { extension at } 72^{\circ} \mathrm{C} \text {. }\end{array}$ & $\begin{array}{l}\text { (LANZ; KUHNERT; BO- } \\
\text { ERLIN, 2003) }\end{array}$ \\
\hline kpc (F) & GTATCGCCGTCTAGTTCTGC & 635 & $\begin{array}{l}5 \mathrm{~min} \text { at } 95^{\circ} \mathrm{C} ; 30 \text { cycles of } 60 \mathrm{~s} \text { at } 95^{\circ} \mathrm{C} \text {, } \\
60 \mathrm{~s} \text { at } 56^{\circ} \mathrm{C}, 60 \mathrm{~s} \text { at } 72^{\circ} \mathrm{C} \text {; and } 5 \mathrm{~min} \text { final } \\
\text { extension at } 72^{\circ} \mathrm{C} \text {. }\end{array}$ & (AZIMI et al., 2013) \\
\hline$n d m(R)$ & $\begin{array}{l}\text { GGTTTGGCGATCTGGTTTTC } \\
\text { CGGAATGGCTCATCACGATC }\end{array}$ & 621 & $\begin{array}{l}10 \mathrm{~min} \text { at } 94^{\circ} \mathrm{C} ; 36 \mathrm{cycles} \text { of } 30 \mathrm{~s} \text { at } 94^{\circ} \mathrm{C} \text {, } \\
40 \mathrm{~s} \text { at } 58^{\circ} \mathrm{C}, 50 \mathrm{~s} \text { at } 72^{\circ} \mathrm{C} \text {; and } 5 \mathrm{~min} \text { final } \\
\text { extension at } 72^{\circ} \mathrm{C} \text {. }\end{array}$ & (POIREL et al., 2011) \\
\hline $\begin{array}{l}\text { tet }-\mathrm{a} \\
\text { (F) } \\
\text { tet - a } \\
\text { (R) }\end{array}$ & AAGCAGGATGTAGCCTGTGC & 372 & $\begin{array}{l}1 \mathrm{~min} \text { at } 94^{\circ} \mathrm{C} ; 30 \text { cycles of } 60 \mathrm{~s} \text { at } 94^{\circ} \mathrm{C}, 60 \\
\mathrm{~s} \text { at } 55^{\circ} \mathrm{C}, 2 \mathrm{~min} \text { at } 72^{\circ} \mathrm{C} \text {; and } 10 \mathrm{~min} \text { final } \\
\text { extension at } 72^{\circ} \mathrm{C} \text {. }\end{array}$ & $\begin{array}{l}\text { (GUILLAUME et al., } \\
\text { 2000) }\end{array}$ \\
\hline $\begin{array}{l}\text { tet }-b \\
\text { (F) } \\
\text { tet }-b(R)\end{array}$ & $\begin{array}{l}\text { GAGACGCAATCGAATTCGG } \\
\text { TTTAGTGGCTATTCTTCCTGCC }\end{array}$ & 228 & $\begin{array}{l}1 \mathrm{~min} \text { at } 94^{\circ} \mathrm{C} ; 30 \text { cycles of } 60 \mathrm{~s} \text { at } 94^{\circ} \mathrm{C}, 60 \\
\mathrm{~s} \text { at } 56^{\circ} \mathrm{C}, 2 \mathrm{~min} \text { at } 72^{\circ} \mathrm{C} \text {; and } 10 \mathrm{~min} \text { final } \\
\text { extension at } 72^{\circ} \mathrm{C}\end{array}$ & $\begin{array}{l}\text { (GUILLAUME et al., } \\
\text { 2000) }\end{array}$ \\
\hline ges-1 to $9,11(F)^{b}$ & AGTCGGCTAGACCGGAAAG & 399 & $\begin{array}{l}10 \mathrm{~min} \text { at } 94^{\circ} \mathrm{C} ; 30 \mathrm{cycles} \text { of } 40 \mathrm{~s} \text { at } 94^{\circ} \mathrm{C} \text {, } \\
40 \mathrm{~s} \text { at } 60^{\circ} \mathrm{C}, 1 \mathrm{~min} \text { at } 72^{\circ} \mathrm{C} \text {; and } 7 \mathrm{~min} \text { final } \\
\text { extension at } 72^{\circ} \mathrm{C}\end{array}$ & $\begin{array}{l}\text { (DALLENNE et al., } \\
\text { 2010) }\end{array}$ \\
\hline per- $1,3(R)^{b}$ & TTCGGCTTGACTCGGCTGA & 520 & $\begin{array}{l}10 \mathrm{~min} \text { at } 94^{\circ} \mathrm{C} ; 30 \mathrm{cycles} \text { of } 40 \mathrm{~s} \text { at } 94^{\circ} \mathrm{C} \text {, } \\
40 \mathrm{~s} \text { at } 60^{\circ} \mathrm{C}, 1 \mathrm{~min} \text { at } 72^{\circ} \mathrm{C} \text {; and } 7 \mathrm{~min} \text { final } \\
\text { extension at } 72^{\circ} \mathrm{C}\end{array}$ & $\begin{array}{l}\text { (DALLENNE et al., } \\
\text { 2010) }\end{array}$ \\
\hline veb $-1,6(R)^{b}$ & CATTTCCCGATGCAAAGCGT & 648 & $\begin{array}{l}10 \text { min at } 94^{\circ} \mathrm{C} ; 30 \text { cycles of } 40 \mathrm{~s} \text { at } 94^{\circ} \mathrm{C} \text {, } \\
40 \mathrm{~s} \text { at } 60^{\circ} \mathrm{C}, 1 \mathrm{~min} \text { at } 72^{\circ} \mathrm{C} \text {; and } 7 \mathrm{~min} \text { final } \\
\text { extension at } 72^{\circ} \mathrm{C}\end{array}$ & $\begin{array}{l}\text { (DALLENNE et al., } \\
\text { 2010) }\end{array}$ \\
\hline
\end{tabular}




\begin{tabular}{|c|c|c|c|c|}
\hline Primer & Primer sequence $\left(5^{\prime}-3^{\prime}\right)$ & $\begin{array}{l}\text { Amplicon } \\
\left(b^{\mathrm{a}}\right)\end{array}$ & PCR Conditions & Reference \\
\hline $\begin{array}{l}\operatorname{imp}(F) \\
\operatorname{imp}(R)\end{array}$ & $\begin{array}{l}\text { GGAATAGAGTGGCTTAATTCTC } \\
\text { CCAAACCACTACGTTATCT }\end{array}$ & 188 & $\begin{array}{l}5 \text { min at } 94^{\circ} \mathrm{C} ; 35 \text { cycles of } 50 \mathrm{~s} \text { at } 94^{\circ} \mathrm{C}, 60 \\
\mathrm{~s} \text { at } 50^{\circ} \mathrm{C}, 1 \mathrm{~min} \text { at } 72^{\circ} \mathrm{C} \text {; and } 8 \text { min final } \\
\text { extension at } 72^{\circ} \mathrm{C}\end{array}$ & $\begin{array}{l}\text { (DALLENNE et al., } \\
\text { 2010) }\end{array}$ \\
\hline $\operatorname{vim}(R)$ & $\begin{array}{l}\text { GATGGTGTTTGGTCGCATA } \\
\text { CGAATGCGCAGCACCAG }\end{array}$ & 390 & $\begin{array}{l}15 \mathrm{~min} \text { at } 95^{\circ} \mathrm{C} ; 36 \text { cycles of } 30 \mathrm{~s} \text { at } 94^{\circ} \mathrm{C} \text {, } \\
20 \mathrm{~s} \text { at } 57^{\circ} \mathrm{C}, 50 \mathrm{~s} \text { at } 72^{\circ} \mathrm{C} \text {; and } 5 \mathrm{~min} \text { final } \\
\text { extension at } 72^{\circ} \mathrm{C} \text {. }\end{array}$ & $\begin{array}{l}\text { (KARUNIAWATI; SA- } \\
\text { HARMAN; LESTARI, } \\
\text { 2013) }\end{array}$ \\
\hline oxa $48(R)$ & CATCAAGTTCAACCCAACCG & 438 & $\begin{array}{l}10 \mathrm{~min} \text { at } 94^{\circ} \mathrm{C} ; 36 \text { cycles of } 30 \mathrm{~s} \text { at } 94^{\circ} \mathrm{C} \text {, } \\
40 \mathrm{~s} \text { at } 54^{\circ} \mathrm{C}, 50 \mathrm{~s} \text { at } 72^{\circ} \mathrm{C} \text {; and } 5 \mathrm{~min} \text { final } \\
\text { extension at } 72^{\circ} \mathrm{C} \text {. }\end{array}$ & (POIREL et al., 2011) \\
\hline uni515 (F) & GCCTCAAGGGCACAACCTCCAAG & 312 & $\begin{array}{l}3 \text { min at } 95^{\circ} \mathrm{C} ; 40 \text { cycles of } 15 \mathrm{~s} \text { at } 95^{\circ} \mathrm{C}, 60 \\
\mathrm{~s} \text { at } 67^{\circ} \mathrm{C}, 01 \mathrm{~min} \text { at } 72^{\circ} \mathrm{C} \text {; and } 5 \mathrm{~min} \text { final } \\
\text { extension at } 72^{\circ} \mathrm{C} \text {. }\end{array}$ & (BARMAN et al., 2008) \\
\hline qacE $\Delta 1(F)$ & CGCAGCGACTTCCACGATGGGGAT & 175 & $\begin{array}{l}5 \text { min at } 94^{\circ} \mathrm{C} ; 30 \text { cycles of } 60 \mathrm{~s} \text { at } 94^{\circ} \mathrm{C}, 60 \\
\mathrm{~s} \text { at } 56^{\circ} \mathrm{C}, 1 \mathrm{~min} \text { at } 72^{\circ} \mathrm{C} \text {; and } 7 \text { min final } \\
\text { extension at } 72^{\circ} \mathrm{C} \text {. }\end{array}$ & (GUO et al., 2015) \\
\hline $\begin{array}{l}\operatorname{ctx}-M(F) \\
\operatorname{ctx}-M(R)\end{array}$ & $\begin{array}{l}\text { ATGTGCAGYACCAGTAARGTKATGGC } \\
\text { TGGGTRAARTARGTSACCAGAAYCAGCGGc }\end{array}$ & 593 & $\begin{array}{l}15 \text { min at } 95^{\circ} \mathrm{C} ; 30 \text { cycles of } 30 \mathrm{~s} \text { at } 94^{\circ} \mathrm{C}, 30 \\
\mathrm{~s} \text { at } 60^{\circ} \mathrm{C}, 2 \mathrm{~min} \text { at } 72^{\circ} \mathrm{C} \text {; and } 10 \mathrm{~min} \text { final } \\
\text { extension at } 72^{\circ} \mathrm{C} \text {. }\end{array}$ & $\begin{array}{l}\text { (LEFLON-GUIBOUT et } \\
\text { al., 2004) }\end{array}$ \\
\hline ctx -M15 (F) & ATAAAACCGGCAGCGGTG & 483 & $\begin{array}{l}5 \mathrm{~min} \text { at } 94^{\circ} \mathrm{C} ; 30 \text { cycles of } 30 \mathrm{sec} \text {. at } 94^{\circ} \mathrm{C} \text {, } \\
30 \mathrm{sec} \text {. at } 55^{\circ} \mathrm{C}, 90 \mathrm{sec} \text {. at } 72^{\circ} \mathrm{C} \text {; and } 7 \mathrm{~min} \\
\text { final extension at } 72^{\circ} \mathrm{C}\end{array}$ & $\begin{array}{l}\text { (LEFLON-GUIBOUT et } \\
\text { al., 2004) }\end{array}$ \\
\hline $\mathrm{ctx}-\mathrm{M} 1(\mathrm{R})$ & AGCTTATTCATCGCCACGTT & 415 & $\begin{array}{l}5 \text { min at } 94^{\circ} \mathrm{C} ; 30 \text { cycles of } 25 \mathrm{sec} \text { at } 94^{\circ} \mathrm{C} \text {; } \\
52^{\circ} \mathrm{C} \text { at } 40 \mathrm{sec} \text {; } 72^{\circ} \mathrm{C} \text { at } 50 \mathrm{sec} ; 6 \text { min final } \\
\text { extension at } 72^{\circ} \mathrm{C}\end{array}$ & $\begin{array}{l}\text { (LEFLON-GUIBOUT et } \\
\text { al., 2004) }\end{array}$ \\
\hline $\operatorname{ctx}-\mathrm{M} 2(\mathrm{R})$ & $\begin{array}{l}\text { CGACGCTACCCCTGCTATT } \\
\text { CCAGCGTCAGATTTTTCAGG }\end{array}$ & 552 & $\begin{array}{l}5 \text { min at } 94^{\circ} \mathrm{C} ; 30 \text { cycles of } 25 \mathrm{sec} \text { at } 94^{\circ} \mathrm{C} \text {; } \\
52^{\circ} \mathrm{C} \text { at } 40 \mathrm{sec} \text {; } 72^{\circ} \mathrm{C} \text { at } 50 \mathrm{sec} ; 6 \text { min final } \\
\text { extension at } 72^{\circ} \mathrm{C}\end{array}$ & $\begin{array}{l}\text { (LEFLON-GUIBOUT et } \\
\text { al., 2004) }\end{array}$ \\
\hline $\operatorname{ctx}-\mathrm{M} 8(\mathrm{R})$ & TCGCGTTAAGCGGATGATGC & 327 & $\begin{array}{l}5 \text { min at } 94^{\circ} \mathrm{C} ; 30 \text { cycles of } 25 \mathrm{sec} \text { at } 94^{\circ} \mathrm{C} \text {; } \\
52^{\circ} \mathrm{C} \text { at } 40 \mathrm{sec} \text {; } 72^{\circ} \mathrm{C} \text { at } 50 \mathrm{sec} ; 6 \mathrm{~min} \text { final } \\
\text { extension at } 72^{\circ} \mathrm{C}\end{array}$ & $\begin{array}{l}\text { (LEFLON-GUIBOUT et } \\
\text { al., 2004) }\end{array}$ \\
\hline ctx - M9 (F) & CAAAGAGAGTGCAACGGATG & 205 & $\begin{array}{l}5 \text { min at } 94^{\circ} \mathrm{C} ; 30 \text { cycles of } 25 \mathrm{sec} \text { at } 94^{\circ} \mathrm{C} \text {; } \\
52^{\circ} \mathrm{C} \text { at } 40 \mathrm{sec} . ; 72^{\circ} \mathrm{C} \text { at } 50 \mathrm{sec} ; 6 \text { min final } \\
\text { extension at } 72^{\circ} \mathrm{C}\end{array}$ & $\begin{array}{l}\text { (LEFLON-GUIBOUT et } \\
\text { al., 2004) }\end{array}$ \\
\hline $\mathrm{ctx}-\mathrm{M} 25(\mathrm{R})$ & AACCCACGATGTGGGTAGC & 327 & $\begin{array}{l}5 \text { min at } 94^{\circ} \mathrm{C} ; 30 \text { cycles of } 25 \mathrm{sec} \text { at } 94^{\circ} \mathrm{C} \text {; } \\
52^{\circ} \mathrm{C} \text { at } 40 \mathrm{sec} \text {; } 72^{\circ} \mathrm{C} \text { at } 50 \mathrm{sec} ; 6 \text { min final } \\
\text { extension at } 72^{\circ} \mathrm{C}\end{array}$ & $\begin{array}{l}\text { (LEFLON-GUIBOUT et } \\
\text { al., 2004) }\end{array}$ \\
\hline mcr-1 (R) & CGCACGATGTGACATTGCTAA & 309 & $\begin{array}{l}15 \mathrm{~min} \text { at } 94^{\circ} \mathrm{C} ; 25 \text { cycles of } \\
30 \text { s at } 94^{\circ} \mathrm{C}, 90 \mathrm{~s} \text { at } 58^{\circ} \mathrm{C}, 60 \mathrm{~s} \text { at } 72^{\circ} \mathrm{C} ; 10 \mathrm{~min} \\
\text { final extension at } 72^{\circ} \mathrm{C} \text {. }\end{array}$ & $\begin{array}{l}\text { (CAVACO; MORD- } \\
\text { HORST; HENDRIKSEN, } \\
\text { 2016) }\end{array}$ \\
\hline
\end{tabular}

a. bp: base pairs;

b. ges, per and veb (multiplex PCR); tem, shv and oxa (multiplex PCR);

c. $S=g$ or $c ; Y=c \operatorname{or} t ; K=g$ or $t ; M=a \operatorname{or} c ; R=g$ or $a$.

Source: This study. 
The PCR products were analyzed by agarose gel electrophoresis in Tris-borate-EDTA buffer. They were stained with Blue-Green Loading Dye I (LGC Biotechnology, São Paulo, Brazil) and visualized under ultraviolet light.

\section{RESULTS}

\section{Sample characteristics}

All 15 cheese samples analyzed were within all parameters established by the research for the sampling process, allowing the results to be more representative of the manufacturing process than the storage or trading. The manufacturing process occurred in three different states from Brazil, divided into 67\% (10/15) from Minas Gerais, 27\% (4/15) from Rio de Janeiro, and 6\% (1/15) from Espírito Santo. The cheeses composition was similar: pasteurized milk, sodium chloride, calcium chloride, lactic acid, and rennet, apart from a sample (sample N) added with lactic acid bacteria and without salt. Thirteen different brands were analyzed.

\section{Phenotypic resistance}

In the pre-selection phase, 98\% (147/150) of pools showed turbidity from microbial growth, indicating a resistant mechanism. Susceptibility $0.2 \%$ (3/150) occurred only in ciprofloxacin tests.

Considering the results from pre-selection and disk diffusion steps, the microbiota from all cheese samples displayed a high phenotypic resistance (CLSI, 2017). Resistance proportions varied from $60 \%$ to $100 \%$ in each cheese. The disc diffusion test revealed that Gram-negative microbiota was resistant to at least eight different antibiotics in $80 \%(12 / 15)$ of the cheese samples and resistant to all tested antibiotics in 13.3\% (2/15) (Figure 1).

Figure 1 - Phenotypic resistance per sample considering the pre-selection and disk diffusion test. In X-axis, cheese samples (A-O). In Y-axis, the number of antibiotics tested.

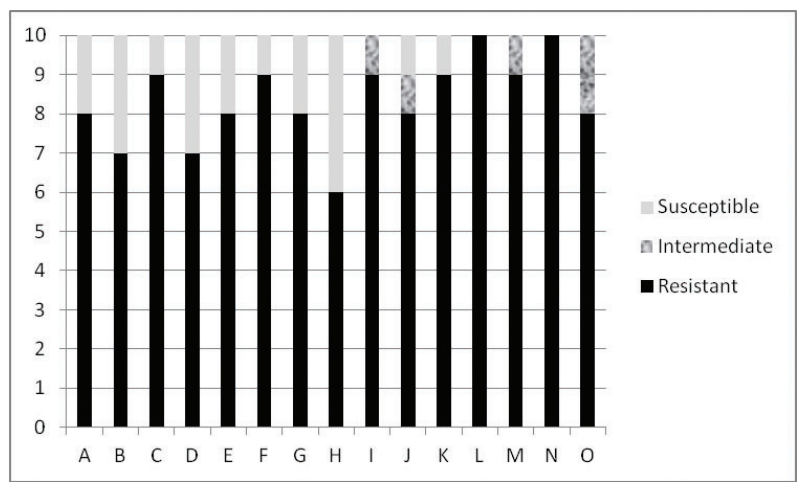

Source: This study.

The 20 susceptible results described in figure 1 include three susceptible from the pre-selection step and seventeen from the disk diffusion step. In total, 130 distinct pools (phenotypically resistant and intermediate) were selected for further DNA extraction and PCR investigation.

Considering the results from the antibiotic's perspective, from ten antibiotics tested, 6 of them showed no susceptibility in 100\% (15/15) of cheese samples. This alarming resistance profile was verified in the chloramphenicol, tetracycline, trimethoprim-sulfamethoxazole, and the beta-lactams ceftazidime, ampicillin, and ampicillin-sulbactam. Even in the remaining four antibiotics tested, the resistance reached high values. Samples were resistant to ertapenem in $86.7 \%$ (13/15) of cheese, $66.7 \%(10 / 15)$ to gentamicin, $46.7 \%$ (7/15) to cefepime (a 4th generation cephalosporin), and 33.3\% (5/15) to ciprofloxacin (fluoroquinolone). Intermediate results or SDD (susceptible dose-dependent), which are therefore dependent on concentration and dose frequency, occurred only in the ciprofloxacin in $6.7 \%(1 / 15)$ and cefepime in $26.7 \%(4 / 15)$.

\section{Comparing breakpoint parameters}

Analyses revealed high rates of resistance according to the CLSI breakpoints. Nevertheless, the resistance indexes would reach even higher rates if the cutoff points of the European Committee on Antimicrobial Susceptibility Testing (EUCAST) were adopted (EUCAST, 2018).

In order to perform an accurate comparison between CLSI and EUCAST, results of resistance tests to tetracycline were not used, since EUCAST does not recommend its use for Enterobacteriaceae. Then, exclusive for this comparison, the maximum number of 135 pools 115 cheese tested with 9 antibiotics) will be considered.

Therefore considering EUCAST, the number of susceptible pools would be reduced by half, representing only $7.4 \%$ (10/135) compared to the CLSI 14.8\% (20/135) susceptible pools in the total. The number of intermediate pools would double, from CLSI 3.7\% (5/135) to 8.8\% (12/135) using EUCAST, and the resistants would reach $83.7 \%$ (113/135) of pools with EUCAST comparing to CLSI $81.5 \%(110 / 135)$ of the pools.

The EUCAST parameter would increase the resistant and intermediate numbers, and decrease the number of susceptible pools to all antibiotics tested. For example, in gentamicin, the $33.3 \%$ susceptible samples would turn into zero according to the EUCAST parameter (Figure 2). Although the two cutoff points are different for all antibiotics tested, samples' resistance results were altered only in four antibiotics, less than half. It is related to the fact that there was no measurable inhibition zone formation in $62.2 \%(84 / 135)$ of the pools. 
Figure 2 - Variations in susceptibility ac cording to CLSI and EUCAST

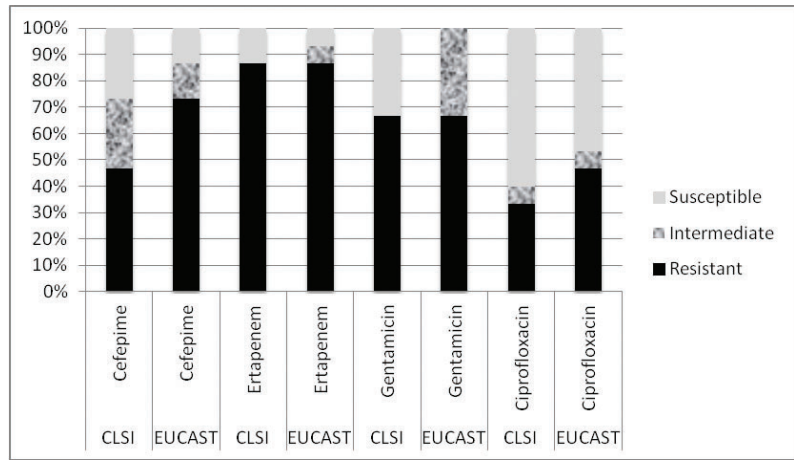

In Y-axis, the percentage value

Source: This study.

\section{Genotypic Resistance}

The PCR assay was performed with multi-species DNA samples. In total, a subset of DNA extracted from 130 pools (resistant or intermediate) was tested for the presence of 25 resistance genes.

Seven different resistance genes were found in $69.2 \%$ (90/130) pools, totalizing 229 genes (Table 2).
Table 2 - Resistance genes quantity in cheese samples and in bacterial pools.

\begin{tabular}{lrr}
\hline Gene & Cheese samples & Quantity in pools \\
\hline int 1 & $93 \%(14 / 15)$ & $48.5 \%(63 / 130)$ \\
tet b & $80 \%(12 / 15)$ & $80.0 \%(12 / 15)$ \\
int 2 & $73 \%(11 / 15)$ & $20.0 \%(26 / 130)$ \\
Shv & $73 \%(11 / 15)$ & $33.1 \%(43 / 130)$ \\
tet a & $73 \%(11 / 15)$ & $73.3 \%(11 / 15)$ \\
ctx- M & $67 \%(10 / 15)$ & $19.2 \%(25 / 130)$ \\
Tem & $67 \%(10 / 15)$ & $34.6 \%(45 / 130)$ \\
ctx- M15 & $6.7 \%(1 / 15)$ & $12.0 \%(3 / 25)$ \\
oxa - 48 & $6.7 \%(1 / 15)$ & $2.4 \%(1 / 84)$ \\
\hline
\end{tabular}

Source: This study.

The numbers of $P C R$ analyses on the quantity in pools column vary according to the resistance presented in each pool, as described in the methodology section.

The genes ges, per, veb, imp, vim, $k p c, n d m$, oxa, oxa48, ctx-M1, ctx-M2, ctx-M8, ctx-M9, ctx-M25, qacE $\Delta 1$, mcr-1 and integron class 3 were not found in the samples.

The most frequently identified gene was the genetic element class 1 integron (int 1), found in 93.3\% (14/15) of cheese samples and $48.5 \%$ (63/130) of the pools. It was found in resistant pools to all antibiotics tested, including an isolate considered intermediate to the fourth-generation cephalosporin.

All cheeses samples harbored at least one of the resistance genes searched. Notably, 80\% (12/15) of cheese presented at least 4 different resistance genes, reaching 7 genes in 20\% (3/15) of cheese samples. Table 3 shows the distribution of a total of 229 genes detected in each pool. In only $30.8 \%(40 / 130)$ of the pools, the genes investigated were not found.

\section{Detection of Enterobacteriaceae family}

The Enterobacteriaceae family was identified by PCR in all 130 positive pools tested. 
Table 3 - Summary of the genes found in each pool.

\begin{tabular}{|c|c|c|c|c|c|c|c|c|c|c|}
\hline Cheese & Cefepime & Ertapenem & Gentamicin & Ampicillin & $\begin{array}{l}\text { Ampicillin- } \\
\text { Sulbactam }\end{array}$ & $\begin{array}{l}\text { Chloram- } \\
\text { phenicol }\end{array}$ & Tetracycline & $\begin{array}{l}\text { Ciproflox- } \\
\text { acin }\end{array}$ & Ceftazidime & $\begin{array}{c}\text { Sulfame- } \\
\text { thoxazol } \\
\text { Trimetho- } \\
\text { prim } \\
\end{array}$ \\
\hline A & & & - & shv, int1, ctx & int 1 & int 1 & ctx & - & & int 1 \\
\hline B & - & - & int1 & shv, int1 & shv, int1 & int1 & & - & & int 1 \\
\hline C & & & $\begin{array}{l}\text { shv, tem, } \\
\text { int1, int2 }\end{array}$ & shv, ctx, int2 & ctx, int2 & shv,int2 & $\begin{array}{l}\text { shv, tem, tet } \\
a \text {, tet } b \text {, int1, } \\
\text { int2 }\end{array}$ & - & int2 & int1 \\
\hline D & - & & - & shv & shv, tem & & $\begin{array}{l}\text { shv, tem, tet } \\
a \text {, tet } b \text {, int2 }\end{array}$ & - & int1 & $\begin{array}{l}\text { shv, tem, } \\
\text { int1, int2 }\end{array}$ \\
\hline$E$ & & & - & & & & tet $b$ & - & & \\
\hline $\mathrm{F}$ & & int1 & - & shv, int1,int2 & $\begin{array}{l}\text { shv, tem, } \\
\text { int1 }\end{array}$ & shv, tem & $\begin{array}{l}\text { shv, tem, tet } \\
\text { a, tet b, int1, } \\
\text { int2 }\end{array}$ & & & ctx, int1 \\
\hline G & - & & ctx, int1 & ctx & ctx, int1 & int1 & $\begin{array}{l}\text { ctx, tet a, tet } \\
b \text {, int2 }\end{array}$ & - & & int1 \\
\hline $\mathrm{H}$ & - & - & - & ctx, int1, int2 & ctx, int1 & & tet a & - & & ctx, int1 \\
\hline I & & & $\begin{array}{l}\text { shv, tem, } \\
\text { int1 }\end{array}$ & $\begin{array}{l}\text { shv, tem, } \\
\text { int1, int2 }\end{array}$ & $\begin{array}{l}\text { shv, tem, } \\
\text { int1 }\end{array}$ & shv & $\begin{array}{l}\text { shv, tem, tet } \\
\text { a, tet b, int1, } \\
\text { int2 }\end{array}$ & & & tem, ctx \\
\hline J & & & ctx, int1 & shv, int1 & ctx & shv, int1 & $\begin{array}{l}\text { shv, tem, tet } \\
\text { a, tet b, int1, } \\
\text { int2 }\end{array}$ & Shv & & ctx, int1, int2 \\
\hline K & & int1 & $\begin{array}{l}\text { shv, tem, } \\
\text { int1, int2 }\end{array}$ & $\begin{array}{l}\text { shv, tem, } \\
\text { int1, int2 }\end{array}$ & $\begin{array}{l}\text { shv, tem, } \\
\text { int1, int2 }\end{array}$ & $\begin{array}{l}\text { shv, tem, } \\
\text { int1, int2 }\end{array}$ & $\begin{array}{l}\text { shv, tem, tet } \\
\text { b, int1, int2 }\end{array}$ & - & & $\begin{array}{l}\text { shv, int1, } \\
\text { int2 }\end{array}$ \\
\hline L & & int1 & tem & tem, int1 & tem, int1 & tem, int1 & $\begin{array}{l}\text { tem, tet } a \text {, } \\
\text { tet } b \text {, int1 }\end{array}$ & tem, int1 & tem & tem, int1 \\
\hline$M$ & & & & shv, ctx, int2 & $\begin{array}{l}\text { shv, tem, } \\
\text { ctx, int2 }\end{array}$ & & $\begin{array}{l}\text { tem, tet } a \text {, } \\
\text { tet } b \text {, int } 2\end{array}$ & tem, int1 & tem & shv, tem \\
\hline$N$ & tem & tem, int1 & tem, int2 & $\begin{array}{l}\text { shv, tem, ctx, } \\
\text { int1 }\end{array}$ & $\begin{array}{l}\text { shv, tem, } \\
\text { ctx, int1 }\end{array}$ & $\begin{array}{l}\text { shv, tem, } \\
\text { int1 }\end{array}$ & $\begin{array}{l}\text { shv, tem, ctx, } \\
\text { tet } a \text {, tet b }\end{array}$ & & & ctx, int1 \\
\hline 0 & int1 & int1 & int1 & $\begin{array}{l}\text { shv, tem, } \\
\text { ct } x-M 15, \\
\text { int1 }\end{array}$ & $\begin{array}{l}\text { shv, tem, } \\
\text { ctx-M15, } \\
\text { int1 }\end{array}$ & $\begin{array}{l}\text { shv, tem, } \\
\text { int1 }\end{array}$ & $\begin{array}{l}\text { shv, tem, tet } \\
a \text {, tet } b \text {, int1 }\end{array}$ & & & $\begin{array}{l}\text { tem, ctx- } \\
-M 15 \text {, shv, } \\
\text { int1 }\end{array}$ \\
\hline
\end{tabular}

Blank frames represent resistant pools, in which the genes tested were not found; (-) represents pools that were not considered phenotypically resistant. Source: This study.

\section{DISCUSSION}

Minas Frescal cheese is a ready-to-eat dairy product, not requiring post-purchase thermal processing, which could contribute to a reduction in its microbiota. In Brazil, it is available for the vulnerable population, mostly consumed by the elderly (EFSA, 2017) and also offered to immunosuppressed patients (VICENSKI; ALBERTI; AMARAL, 2012).

Evaluation of antibiotic resistant microbiota, although not usually researched in food, can convey more complete data than those obtained with isolation techniques, corroborated by metagenomics research presenting more than 500 genera in a ripened cheese (ESCOBAR-ZEPEDA; SANCHEZ-FLORES; QUIRASCO BARUCH, 2016).

\section{Phenotypic Resistance}

So far, to our knowledge, this study presents the first phenotypic identification of resistance to ertapenem in
Minas Frescal cheese in Brazil. The percentage of $86.7 \%$ of resistance found in a ready-to-eat food is a high rate, especially considering that Enterobacteriaceae family resistant to carbapenems are ranked as a priority group to development and research of new antibiotics according to the global priority pathogens list (WHO, 2017). Another group established as critic is the Enterobacteriaceae resistant to the third-generation cephalosporin. In this paper, this resistance reached $100 \%$ (15/15) of the cheese samples, a high percentage when compared to the 35\% found in soft cheese by Omoshaba et al., 2018. It is noteworthy that, even in cephalosporin of fourth-generation resistance was observed in $46.7 \%$ of cheese samples.

It is alarming that besides the ceftazidime, five different antibiotics, namely, ampicillin, ampicillin-sulbactam, chloramphenicol, tetracycline, and trimethoprim-sulfamethoxazole, demonstrated no susceptibility in all different cheese 
samples from 13 different brands. Values are higher than those found in multidrug-resistant enterotoxigenic Staphylococcus aureus isolated from milk tanks (PEXARA et al., 2016).

\section{Comparing parameters}

The startling growth (of the majority of the pools) to the limit of detection - $6 \mathrm{~mm}$ (the disk diameter), allows only the categorization as resistant, irrespective of the cut off applied, which emphasizes the high prevalence of resistance found in cheese microbiota.

Among the pools in which the difference between the two parameters could be evaluated, there was a significant reduction in the number of susceptible pools and an increasing in the resistant and intermediate by applying the EUCAST criterion as it has more stringent breakpoints for susceptibility, though less widespread than the CLSI standards. In other study that difference proved to be less clear, although the EUCAST parameter is slightly more rigid (KASSIM et al., 2016).

\section{Genotypic Resistance}

Corroborating the high phenotypic resistance found, $100 \%$ of cheese samples harbored at least one gene investigated, reaching up to the co-existence of 7 different genes on the same sample.

\section{Integrons}

The integrons are genetic elements that have a key role in the adaptation of bacteria to antibiotic therapy. The cells that contain integrons can select in a pool of genes and fix some genes under intense selective pressure imposed by the use of antibiotics in human medicine, veterinary, and agriculture (GILLINGS, 2014).

The most studied and identified integron in foods, as well as in other areas, is the class 1 integron, having their cassettes more fully described in the literature (GILLINGS, 2014). This ubiquitous characteristic may explain its presence in an SDD pool to a broad-spectrum antibiotic, the cefepime (fourth-generation cephalosporin).

In samples analyzed, the class 1 integron was the most prevalent. The values found in this article are even higher than those found in a study with metagenomics approach, where $77 \%$ of 35 Minas Frescal cheese presented class 1 integron (DE PAULA et al., 2018).

Despite the recognized importance in the clinical area, the class 2 integrons are still poorly surveyed in the food sector. In our study, it was detected in 73\% (11/15) of samples, particularly prevalent in tetracycline-resistant pools, which are commonly associated with int 1 (BELAYNEHE; SHIN; YOO, 2018). An alarming amount of 97\% has already been found in a metagenomic study with 35 Minas Frescal cheese samples (DE PAULA et al., 2018).

Research of integron class 3 in food is rare. In our research, none of the cheese samples examined pre- sented class 3 integron likewise in a similar study with cheese samples (DE PAULA et al., 2018)

Though the integrons research is still scarce in the food sector and focused on a small number of bacterial species or class of integron (GILLINGS, 2014), our analysis revealed that more than half (56.2\%) of the pools possessed one integron system, and even two systems simultaneously in the same pool which reinforces the importance of investigate these genetic systems to understand its function in food safety.

\section{Tetracycline resistant genes}

In our study, $100 \%$ of the samples were categorized as phenotypically tetracycline resistant. The high phenotypic resistance and co-existence of tet $a$ and tet $b$ in $66.7 \%$ (10/15) of samples may be related to the high rates of integrons found (BELAYNEHE; SHIN; YOO, 2018).

Comparable values were found in Minas Frescal cheese varying from $15 \%$ to $100 \%$ of prevalence of tet genes (DE PAULA et al., 2018). Lower values were found even in raw milk Egyptian cheese in which it was found in 5\% of the isolates (HAMMAD; HASSAN; SHIMAMOTO, 2015).

Study on the use of antibiotics in cattle herds in Minas Gerais State, the State where most of the samples of this study are manufactured, identified that tetracycline and aminoglycosides were the most frequent antibiotics used against mastitis (VIEIRA et al., 2016). This is particularly crucial in the case of cheese, a product of animal origin. Evidence shows that interventions that restrict the use of antibiotics in food-producing animals are associated with a reduction in the presence of antibiotic-resistant bacteria in those animals (TANG et al., 2017).

\section{Beta-lactamase-producing genes}

Most extended-spectrum beta-lactamases (ESBLS) evolved from genetic mutations in beta-lactamases tem and shv types and are considered an important mechanism that confers resistance to penicillins and cephalosporins, including the third and fourth generation (DALLENNE et al., 2010).

In our analysis, the most frequent ESBL encoding gene was the shv, present in $73.3 \%$ of samples. The shv gene was identified in 6 different antibiotic-resistant pools mainly among the penicillins but either among the non-beta-lactams: tetracycline and chloramphenicol. Multiple drug resistance has been commonly observed in the majority of ESBL-producing, and alarmingly the co-resistance to other antibiotics in common use, such as aminoglycosides, fluoroquinolones, and tetracycline has been frequently reported (XU et al., 2015).

The highest count of tem genes was found in tetracycline's resistant pools, followed by the penicillins. The tem gene rates (67\% of the samples) were comparable with a metagenomics study in Minas Frescal cheese, present in 91.4\% of the samples but without phenotypic evaluation (DE PAULA et al., 2018). 
The ESBLs ctx family that acts in cephalosporins has been identified in Brazil, predominantly in clinical samples, especially in Klebsiella pneumoniae and Escherichia coli (ROCHA; PINTO; BARBOSA, 2016). In our study, the ctx gene was found in $63.3 \%$ of samples, mainly among the penicillins-beta-lactams.

Important to highlight that in the 72 pools with ESBLS encoding genes found in this research, 32 presented simultaneously at least two of beta-lactamase (bla) genes described (tem, shv, and ctx). Although the bla genes tem, ctx, and shv genes have already been found in E. coli isolated from cheese and milk, it was restricted to ampicillin-resistant pools and in lower proportions $(18.0 \%$, $4.0 \%$, and $1.4 \%$ respectively) (OMBARAK et al., 2018). The values found in this paper are superior, even compared to the ESBL-encoding genes researched in E. coli isolated from mastitic milk (EISENBERGER et al., 2018).

The bla genes ges, per, veb, $k p c, n d m$, imp, vim, oxa, and oxa-48 were not found in samples analyzed. These genes are not commonly investigated in cheese or even dairy products.

\section{Quaternary ammonium resistance}

Quaternary ammonium is a class of cationic surfactants present in disinfectants, biocides, and detergents used intensively in hospitals and industry. The resistance genes qac can be carried on class 1 integron what increases the co-selection of antibiotic resistance (ZHANG et al., 2015). Although the high prevalence rates of int 1 in this paper, the qac $\triangle E 1$ gene was not found.

\section{Polymyxin E resistance}

The mcr-1 gene that evaluates the genotypic resistance to polymyxin E mediated by plasmid was not found.

\section{CONCLUSION}

So far, to our knowledge, this is the first phenotypic identification of a microbiota resistant to ertapenem in Minas Frescal cheese in Brazil.

The amount and diversity of genes found, particularly the integrons, with a methodology that evaluates the microbiota point out the food area as an important sector to be managed to reduce the process of antibiotic resistance.

\section{Data availability}

The datasets of the microorganisms analyzed during the current study are available in the Coleção de Cultura de Bactérias Resistentes de Origem Alimentar-CCBROA, available in Sistema de Informação sobre a Biodiversidade Brasileira - SiBBr (https://ipt.sibbr.gov.br/sibbr/ resource? $r=c c b r o a)$.

A portion of the microorganisms harboring resistance genes identified by PCR in this research had the DNA sequenced and will be published posteriorly.

\section{Acknowledgments}

The authors are grateful to the research funding support from the Federal University of the State of Rio de Janeiro (UNIRIO).

\section{Funding}

This work was supported by Federal University of the State of Rio de Janeiro (UNIRIO).

\section{REFERENCES}

AZIMI, L. et al. Evaluation of phenotypic methods for detection of klebsiella pneumoniae carbapenemase-producing $\mathrm{k}$. pneumoniae in tehran. J. Med. Bacteriol., Tehran, v. 2, n. 3, p. 26-31, 2013. Disponivel em: https://www.semanticscholar.org/paper/Evaluationof-Phenotypic-Methods-for-Detection-of-Azimi-Rastegarlari/25e8de 806d4a5edb1792b507967c7dd84f7a105c. Acesso em: 5 Feb. 2018.

BARMAN, M. et al. Enteric salmonellosis disrupts the microbial ecology of the murine gastrointestinal tract. Infect. Immun., Washington, v. 76, n. 3, p. 907-915, 2008. Disponível em: http://www.ncbi.nlm. nih.gov/pubmed/18160481. Acesso em: 5 Feb. 2018. DOI: 10.1128/ IAI.01432-07.

BELAYNEHE, K. M.; SHIN, S. W.; YOO, H. S. Interrelationship between tetracycline resistance determinants, phylogenetic group affiliation and carriage of class 1 integrons in commensal Escherichia coli isolates from cattle farms. BMC Veterinary Research, London, v. 14, n. 1, p. 340, 2018. Disponível em: https://bmcvetres.biomedcentral.com/ articles/10.1186/s12917-018-1661-3. Acesso em: 5 Feb. 2018. DOI: 10.1186/s12917-018-1661-3.

BRASIL. Ministério da Agricultura, Pecuária e Desenvolvimento. Portaria $\mathrm{n}^{\circ}$ 352, de 4 de setembro de 1997 [Regulamento técnico para fixação de identidade e qualidade do queijo Minas Frescal]. Diário Oficial [da] União, Poder Executivo, Brasília, DF, 08 de setembro de 1997, Brasília, 1997. Disponível em: http://extranet.agricultura.gov. $\mathrm{br} /$ sislegis-consulta/servlet/VisualizarAnexo $? \mathrm{id}=12874$. Acesso em: 21 Dec. 2017.

CAVACO, L.; MORDHORST, H.; HENDRIKSEN, R. Laboratory protocol: PCR for plasmid-mediated colistin resistance genes, mcr-1 and mcr-2 (multiplex). DTU food national food institute, Lyngby, v. 2, n. 1016 , p. 1-15, 2016. Disponível em: https://www.eurl-ar.eu/CustomerData/ Files/Folders/21-protocols/278_mcr-multiplex-pcr-protocol-v2-oct16. pdf. Acesso em: 5 Feb. 2018.

CDC. Laboratory protocol for detection of carbapenem-resistant or carbapenemase-producing, klebsiella spp . and escrerichia coli from rectal swabs. Centro de Controle e Prevenção de Doenças, Atlanta, n. 5, p. 1-6, 2008. Disponível em: https://www.cdc.gov/hai/pdfs/ labsettings/klebsiella_or_ecoli.pdf. Acesso em: 9 Jan. 2018.

CLSI. M 100 Performance standards for antimicrobial susceptibility testing. Clinical and Laboratory Standards Institute, Wayne, v. 27, p. 32-42, 2017. Disponível em: http://www.facm.ucl.ac.be/intranet/CLSI/ CLSI-2017-M100-S27.pdf. Acesso em: 13 Jan. 2018.

DALLENNE, C. et al. Development of a set of multiplex PCR assays for the detection of genes encoding important $\beta$-lactamases in Enterobacteriaceae. Journal of Antimicrobial Chemotherapy, London, v. 65 , n. 3, p. 490-495, 2010. DOI: 10.1093/jac/dkp498. Disponível em: https://academic.oup.com/jac/article-lookup/doi/10.1093/jac/ dkp498. Acesso em: 9 Jan. 2018.

DE PAULA, A. C. et al. Antibiotic resistance genetic markers and integrons in white soft cheese: aspects of clinical resistome and potentiality of horizontal gene transfer. Genes, Basel, v. 9, n. 2, p. 106, 2018. Disponível em: http://www.mdpi.com/2073-4425/9/2/106. 
Acesso em: 26 June 2018. DOI: 10.3390/genes9020106.

EFSA. A European one health action plan against antimicrobial resistance (AMR). European Food Safety Authority, Parma, p. 24, 2017. Disponível em: https://ec.europa.eu/health/amr/sites/amr/files/ amr_action_plan_2017_en.pdf. Acesso em: 15 jan. 2018.

EISENBERGER, D. et al. Molecular characterization of extendedspectrum $\beta$-lactamase-producing escherichia coli isolates from milk samples of dairy cows with mastitis in bavaria, Germany. Microbial Drug Resistance, Larchmont, v. 24, n. 4, p. 505-510, 2018. Disponível em: http://online.liebertpub.com/doi/10.1089/mdr.2017.0182. Acesso em: 5 fev. 2018. DOI: 10.1089/mdr.2017.0182.

ESCOBAR-ZEPEDA, A.; SANCHEZ-FLORES, A.; QUIRASCO BARUCH, M. Metagenomic analysis of a Mexican ripened cheese reveals a unique complex microbiota. Food Microbiology, London, v. 57, p. 116-127, 2016. DOI: 10.1016/j.fm.2016.02.004. Disponível em: http://www. sciencedirect.com/science/article/pii/S0740002016000113. Acesso em: 4J an. 2018.

EUCAST. European Committee on Antimicrobial Susceptibility Testing Breakpoint tables for interpretation of MICs and zone diameters. European Society of Clinical Microbiology and Infectious Diseases, Basel, v. 8.1, p. 5-9, 2018. Disponível em: http://www.eucast.org/ fileadmin/src/media/PDFs/EUCAST_files/Breakpoint_tables/v_8.1_ Breakpoint_Tables.pdf. Acesso em: 27 June 2018.

GILLINGS, M. R. Integrons: Past, Present, and Future. Microbiology and Molecular Biology Reviews, Washington, v. 78, n. 2, p. 257-277, 2014. Disponível em: http://www.ncbi.nlm.nih.gov/pubmed/24847022. Acesso em: 18 jan. 2018. DOI: 10.1128/MMBR.00056-13.

GUILLAUME, G. et al. PCR typing of tetracycline resistance determinants (Tet A-E) in Salmonella enterica serotype Hadar and in the microbial community of activated sludges from hospital and urban wastewater treatment facilities in Belgium. FEMS Microbiology Ecology, Amsterdam, v. 32, n. 1, p. 77-85, 2000. Disponível em: https://academic.oup.com/femsec/article-lookup/ doi/10.1111/j.1574-6941.2000.tb00701.x. Acesso em: 9 Jan. 2018. DOI: 10.1111/j.1574-6941.2000.tb00701.x.

GUO, L. et al. Antimicrobial and disinfectant resistance of Escherichia coli isolated from giant pandas. Journal of Applied Microbiology, Oxford, v. 119, n. 1, p. 55-64, 2015. DOI: 10.1111/jam.12820. Disponível em: http://doi.wiley.com/10.1111/jam.12820. Acesso em: 5 Feb. 2018.

HAMMAD, A.M.; HASSAN, H. A.; SHIMAMOTO, T. Prevalence, antibiotic resistance and virulence of Enterococcus spp. in Egyptian fresh raw milk cheese. Food Control, Vurrey, v. 50, p. 815-820, 2015. Disponível em: http://linkinghub.elsevier.com/retrieve/pii/S0956713514006021. Acesso em: 5 Feb. 2018. DOI: 10.1016/j.foodcont.2014.10.020.

INSTITUTO BRASILEIRO DE GEOGRAFIA E ESTATÍSTICA (IBGE). Pesquisa de Orçamentos Familiares 2008-2009. Rio de Janeiro: IBGE, 2010. p. 130. Disponível em: https://biblioteca.ibge.gov.br/visualizacao/livros/ liv45419.pdf. Acesso em: 4 Jan. 2018.

KARUNIAWATI, A.; SAHARMAN, Y.; LESTARI, D. Detection of carbapenemase encoding genes in enterobacteriace, pseudomonas aeruginosa, and acinetobacter baumanii isolated from patients at intensive care unit cipto mangunkusumo hospital in 2011. Acta medica Indonesiana, Jakarta, v. 45, p. 101-106, 2013. Disponível em: https:// www.ncbi.nlm.nih.gov/pubmed/23770789. Acesso em: 5 Feb. 2018.

KASSIM, A. et al. Comparison of Clinical Laboratory Standards Institute and European Committee on Antimicrobial Susceptibility Testing guidelines for the interpretation of antibiotic susceptibility at a University teaching hospital in Nairobi, Kenya: a cross-sectional stud. Annals of Clinical Microbiology and Antimicrobials, London, v. 15, n. 1, p. 21, 2016.. Disponível em: http://ann-clinmicrob.biomedcentral. com/articles/10.1186/s12941-016-0135-3. Acesso em: 24 June 2018. DOI: $10.1186 / \mathrm{s} 12941-016-0135-3$

LANZ, R.; KUHNERT, P.; BOERLIN, P. Antimicrobial resistance and resistance gene determinants in clinical Escherichia coli from different animal species in Switzerland. Veterinary Microbiology, Amsterdam, v. 91, n. 1, p. 73-84, 2003. Disponível em: http://www.sciencedirect. com/science/article/pii/S0378113502002638. Acesso em: 9 Jan. 2018. DOI: 10.1016/S0378-1135(02)00263-8.

LEFLON-GUIBOUT, V. et al. Emergence and spread of three clonally related virulent isolates of CTX-M-15-producing escherichia coli with variable resistance to aminoglycosides and tetracycline in a french geriatric hospital. Antimicrobial Agents and Chemotherapy, Washington, v. 48, n. 10, p. 3736-3742, 2004. Disponível em: http:// www.ncbi.nlm.nih.gov/pubmed/15388428. Acesso em: 29 June 2018. DOI: 10.1128/AAC.48.10.3736-3742.2004.

OMBARAK, R. A. et al. Prevalence and molecular characterization of antimicrobial resistance in escherichia coli isolated from raw milk and raw milk cheese in Egypt. Journal of Food Protection, Des Moines, v. 81, n. 2, p. 226-232, 2018. Disponível em: http://jfoodprotection.org/ doi/10.4315/0362-028X.JFP-17-277. Acesso em: 5 Feb. 2018. DOI: 10.4315/0362-028X.JFP-17-277.

OMOSHABA, E. O. et al. Prevalence and antibiotic resistance patterns of methicillin-resistant Staphylococcus aureus in raw milk and soft cheese (wara) sold in Abeokuta, Nigeria. Sokoto Journal of Veterinary Sciences, Sokoto, v. 16, n. 1, p. 1, 2018. Disponível em: https://www. ajol.info/index.php/sokjvs/article/view/168039. Acesso em: 27 June 2018. DOI: 10.4314/sokjvs.v16i1.1.

PEXARA, A. et al. Occurrence and antibiotic resistance of enterotoxigenic Staphylococcus aureus in raw ovine and caprine milk in Greece. Dairy Science \& Technology, Paris, v. 96, n. 3, p. 345-357, 2016. Disponível em: http://link.springer.com/10.1007/s13594-015-0272-z. Acesso em: 28 Dec. 2017. DOI: 10.1007/s13594-015-0272-z.

POIREL, L. et al. Detection of NDM-1-Producing Klebsiella pneumoniae in Kenya. Antimicrobial Agents and Chemotherapy, Washington, v. 55, n. 2, p. 934-936, 2011. Disponível em: http://www.ncbi.nlm. nih.gov/pubmed/21115785. Acesso em: 9 Jan. 2018. DOI: 10.1128/ AAC.01247-10

ROCHA, F. R.; PINTO, V. P. T.; BARBOSA, F. C. B. The Spread of CTXM-Type Extended-Spectrum $\beta$-Lactamases in Brazil: A Systematic Review. Microbial Drug Resistance, Larchmont, v. 22, n. 4, p. 301-311, 2016. Disponível em: http://online.liebertpub.com/doi/10.1089/ mdr.2015.0180. Acesso em: 5 Feb. 2018. DOI: 10.1089/mdr.2015.0180.

TANG, K. L. et al. Restricting the use of antibiotics in food-producing animals and its associations with antibiotic resistance in foodproducing animals and human beings: a systematic review and meta-analysis. The Lancet Planetary Health, Netherlands, v. 1, n. 8, p. e316-e327, 2017. Disponível em: http://www.thelancet.com/pdfs/ journals/lanplh/PIIS2542-5196(17)30141-9.pdf. Acesso em: 2 Dec. 2017. DOI: 10.1016/S2542-5196(17)30141-9

VICENSKI, P. P.; ALBERTI, P.; AMARAL, D. J. C. do. Dietary recommendations for immunosuppressed patients of 17 hematopoietic stem cell transplantation centers in Brazil. Revista Brasileira de Hematologia e Hemoterapia, São Paulo, v. 34, n. 2, p. 86-93, 2012. Disponível em: http://www.pubmedcentral.nih.gov/articlerender.fcg i? artid=3459399\&tool=pmcentrez\&rendertype=abstract. Acesso em: 5 Feb. 2018. DOI: 10.5581/1516-8484.20120028.

VIEIRA, V. A. et al. Práticas de uso de antimicrobianos em rebanhos bovinos de unidades de agricultura familiar no Norte de Minas Gerais. Caderno de Ciências Agrárias, Montes Claros, v. 8, n. 1, p. 8-15, 2016. Disponível em: https://periodicos.ufmg.br/index.php/ccaufmg/article/ view/2806. Acesso em: 5 Feb. 2018. 
WORLD HEALTH ORGANIZATION (WHO). Global priority list of antibioticresistant bacteria to guide research, discovery, and development of new antibiotics. Genebra: WHO, 2017. p. 7. Disponível em: http://www. who.int/medicines/publications/WHO-PPL-Short_Summary_25FebET_NM_WHO.pdf. Acesso em: 4 Jan. 2018.

$\mathrm{XU}, \mathrm{G}$. et al. Prevalence and characteristics of extended-spectrum $\beta$-lactamase genes in Escherichia coli isolated from piglets with post-weaning diarrhea in Heilongjiang province, China. Frontiers in Microbiology, Lausanne, v. 6, p. 1103, 2015. Disponível em: http:// www.ncbi.nlm.nih.gov/pubmed/26500640. Acesso em: 7 Dec. 2017. DOI: 10.3389/fmicb.2015.01103.

ZHANG, C. et al. Quaternary ammonium compounds (QACs): A review on occurrence, fate and toxicity in the environment. Science of The Total Environment, Amsterdam, v. 518/519, p. 352-362, 2015. Disponível em: https://www.sciencedirect.com/science/article/pii/ S0048969715002727. Acesso em: 29 June 2018. DOI: 10.1016/j. scitotenv.2015.03.007.

Submetido em: 08/02/2020

Aceito em: 16/09/2020 